\title{
Increasing Production of Engineered Cells in Multiple Myeloma Using Microfluidic Technology
}

\author{
arezoo karamivandishi ${ }^{1}$, Masoud Soleimani ${ }^{1}$, and mina soufizomorrod ${ }^{1}$ \\ ${ }^{1}$ Tarbiat Modares University Faculty of Medical Sciences
}

June 9, 2020

\begin{abstract}
The potential use of gene- modified cell therapy in hematologic malignancies is often limited by complications related to effectively engineering and manufacturing cells with conventional delivery systems and is challenge specifically for immune cells. In fact, this life-saving therapy requires use of inefficient reagents and specialized equipment that can drive up the price of the treatment. Herein, we compared two different approaches for gene transfer into target cells: Nucleofection as a 2D gene delivery and microfluidic device as a 3D gene transfer technology. In fact, we developed and used serpentine microfluidic chip for cell membrane penetration that permits delivery of DNA into Multiple myeloma cells. We achieved high transfection efficiency (55.7\% GFP) in myeloma cells with high cell viability (by PI staining) 24-48 hours after microfluidic processing compared to nucleofection that is toxic and rate of dead cells is very high. The significant differences in outcomes from the two techniques underscores the importance of understanding the impact of intracellular delivery techniques on cell function for research and clinical applications. Altogether, these results highlight the use of microfluidic device as a rapid and gentle delivery method with promising potential to engineer primary human cells for research and clinical applications.
\end{abstract}

\section{Keywords}

Multiple Myeloma, Nucleofection, Microfluidic Device, Gene Delivery

\section{Introduction}

Multiple myeloma is a differentiated plasma cell malignancy and is the most common hematological malignancy after non-Hodgkin's Lymphoma (Siegel, Miller, \& Jemal, 2016). This disorder accounts for 1\% of all malignancies and $10 \%$ of blood malignancies. The disease is characterized by the clonal proliferation of malignant plasma cells in the bone marrow, which results in the overproduction of monoclonal immunoglobulins in the blood and urine of patients. In addition, patients develop significant bone lesions that endanger patients' quality and survival time (Kyle et al., 2003)(Palumbo \& Anderson, 2011). Multiple myeloma has been an obstructive disease to treat. The median overall survival was approximately 3 years until the late 1990s. The introduction of high-dose therapy, particularly, and autologous stem cell transplantation, as well as the development of new drugs, such as immunomodulatory drugs (IMiDs) and proteasome inhibitors lead to the improvement in survival. However, finally most MM patients relapse which reveal that there is a need for novel treatment approaches (Franssen, Mutis, Lokhorst, \& van de Donk, 2019).

Ex vivo manipulation of cells is crucial for the success of cell-based therapies, such as chimeric antigen receptor $\mathrm{T}$ cells for the treatment of cancers but challenges in delivery technologies have limited this approach (Cox, Platt, \& Zhang, 2015).Current method for manufacturing gene-modified cells requires the use of viruses which is time consuming and expensive (Wang \& Rivière, 2016). In addition, viral gene delivery system needs safety and monitoring of patients after treatment (Levine, Miskin, Wonnacott, \& Keir, 2017).non-viral gene delivery is an alternative to viral- based gene modified cell therapy(GMCT) because of low cost, easy to scale and brief lead time. Nucleofection is one of the routine non-viral techniques that can transfer genetic 
materials to broad range of cells. This method is easy, rapid, efficient that resulted in greater than $80 \%$ expression of transferred constructs in human T cells (Tchou et al., 2017). However, because of reduced cell viability and disruptions on global gene expression, cytokine production, lineage markers, and in vivo function of cells, now is less than ideal for GMCT (Escoffre et al., 2009)'(Rosazza, Haberl Meglic, Zumbusch, Rols, \& Miklavcic, 2016). Thus, novel non-viral intracellular delivery strategies are required to overcome limitations of conventional methods in efficient and nontoxic transfer of various macromolecules to the immune cell engineering.

Microfluidic technology is the science of using low amounts of liquid flow (microliter, nanoliter) in the synthesized microsystems. The applications of this approach in biology and medicine have led to the creation of a new research direction. Since the beginning of this technology, in the 1990's, almost concomitant with the advent of micro-scale chemistry techniques, microfluidics have grown rapidly and persisted with the promise of evolving conventional laboratory analysis techniques (Loessner et al., 2010).Benefits of this technique include significant reduction in material and sample consumption, increased speed and sensitivity to reactions, separation and detection, reduced cost and time of operation, and importantly in the threedimensional imitation of cell environment (Whitesides, 2006). Since gene transfer with conventional systems requires complex functional processes and lacks the sensitivity and specificity as well as low viability and efficiency, rapid advancement in the field of microfluidic knowledge opens new window for gene transfer and Gene therapy .In general, the microfluidic method has facilitated the transfer of macromolecules into the cell by scaling the channel and cell dimensions(Xia et al., 2010).

Since intracellular transfer of biomolecules such as DNA and siRNA to immune cells is difficult, so here, we describe the development and use of our user-friendly microfluidic transfection device for hydro dynamically intracellular delivery of DNA into myeloma cells using serpentine channels. we delivered about $11.0 \mathrm{~Kb}$ DNA construct expressing green fluorescent protein (GFP) and anti-BCMA marker into human Myeloma cell and showed high levels of cell viability, and gene expression. This work provides comparative functional consequences of nucleofection as a 2D gene transfer strategy relative to an innovative mechanical delivery strategy as 3D gene delivery approach.

\section{Materials and Methods}

\section{Cell culturing}

RPMI-8226 Cells were grown in RPMI 1640 medium supplemented with 10\% FBS and 1\% L-glutamine. Cells were cultured at $37^{\circ} \mathrm{C}$ in the presence of $5 \% \mathrm{CO} 2$.

\section{Nucleofection}

cells were transfected using the Amaxa nucleofector II device (Lonza). Cells were re suspended in specific solutions. Briefly, according to amaxa guidelines , $2.5 \times 10^{6}$ RPMI- 8226 cells were re suspended in $100 \mu l$ of Nucleofection buffer solution containing $5 \mu \mathrm{g}$ DNA, and the cells were transfected using Program G-016 of the Nucleofector II and immediately transferred into 6-well plates containing $37{ }^{\circ} \mathrm{C}$ pre-warmed culture medium

Device Design and FabricationMold was fabricated using conventional photolithography techniques. SU82550 negative photoresist was spin coated on silicon wafer and baked. PDMS pre-polymer and the cross linker were mixed at the recommended $10: 1 \mathrm{w} / \mathrm{w}$ ratio. After pouring the mixture onto the SU-8 master and degassing for $1 \mathrm{~h}$, the mixture was cured at $75 \mathrm{C}$ for $2 \mathrm{~h}$. PDMS moldings were cut and peeled off the masters. The main components of such devices are: (i) inlet and outlet (ii) main channel: a serpentine-like channel, $100 \mu \mathrm{m}$ width and $60 \mu \mathrm{m}$ height, consisting of turns with length of $12 \mathrm{~cm}$.

\section{Gene delivery into cells via microfluidic device}

To examine the potential of the fabricated design to enable intracellular delivery, target cells were centrifuged at $1200 \mathrm{rpm}$ for 5 minutes. Cell pellets were dissolved in 5\% serum containing medium. Myeloma cells and plasmids were introduced into the microfluidic transfection device either in tandem or sequentially by flowing 
suspension medium. Various velocities were investigated to generate chaotic advection in the device. The speed was set at a range of 3-13 ml / hr. After completion of process, the cells were Collected and suspended in appropriate culture media and plated in appropriately sized standard culture plates at $2 \times 106 \mathrm{cells} / \mathrm{ml}$ which incubated in a $37^{\circ} \mathrm{C}$ incubator with $5 \% \mathrm{CO} 2$.

Flow CytometryTo analyze the percentage of GFP positive cells by BD FACS Calibur Flow Cytometer, PE-anti BCMA antibody (407107) was purchased from Biolegend. Cells for flow analysis were suspended in flow buffer (PBS with 0.5\% BSA) containing antibody and incubated for $15 \mathrm{~min}$ at room temperature.

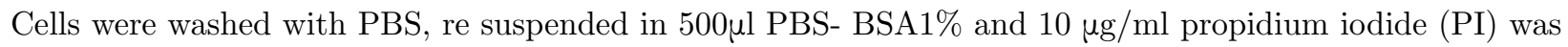
added immediately before flow cytometry analysis. The transfection efficiency was determined $24-48$ hours post transfection.

Statistical AnalysisStatistical analysis was performed using GraphPad Prism 6 and FlowJo. All data are represented as means $\pm \mathrm{SD}$.

\section{Results}

\section{Verification of microfluidic transfection device}

Chaotic advection is a hydrodynamic phenomenon in which enhances stretching and folding of particle interfaces. This modification of fluid-fluid boundaries augments interfacial area (Aref, 1984).Increasing mixing and diffusion is of special importance in flows containing large biomolecules and cells. However, flow in small length-scale microfluidic device is generally laminar with low Reynolds number but special-designed device can lead to spatial advection which cause enhanced agitation in micrometer dimensions. Producing chaotic motion across the cell surface will enable each target plasmid to eventually visit every cell, which will increase the probability of target DNAs that impinge on cell membrane and/or reduce the required amount of target biomolecules (fig. 1A,1B). In this study, we hypothesize that serpentine device can be used to transfect target cells under continuous perfusion.

To determine if hydrodynamic conditions required to induce and sustain chaos in our flow cells, we observed and characterized flow dynamics using computational fluid dynamic simulations. Computational fluid dynamic simulations were used to determine the velocity magnitude and direction of induced chaotic advection in our flow cell (fig.1C). In fact, velocity in inner wall of microfluidic chip is higher than outer wall that can enhances entrance of DNA to cell.

\section{Increased delivery efficiency with high cell viability in serpentine device}

Nucleofection and viral transduction are effective methods for intracellular delivery of gene into different cells (Bilal, Vacaflores, \& Houtman, 2015)'(Kebriaei et al., 2016) but because of substantial reduction in cell viability and recovery of cells using this conventional transfection methods, we sought to investigate if comparable intracellular delivery efficiencies and higher viability could be achieved via our pattern.

For our proof-of-concept study, we selected an approximately $11.0 \mathrm{kB}$ GFP construct including anti-BCMA gene to transfect into myeloma cell via serpentine microfluidic device. We observed an increased level of GFP expression compared to cells processed through nucleofection. (Fig. 2)

To confirm that lower DNA volumes in our pattern is required compared to nucleofection, various plasmid concentration was applied to the device. Also, the effects of different speeds were evaluated on the percentage of GFP positive cells as shown in the diagram (fig 3). Our data showed that delivery efficiency increased with increasing flow rate across designed pattern but viability in flow rate of $13 \mathrm{ml} / \mathrm{h}$ decreased compared to lower speeds. However, viability impacts on expression of critical genes associated with function, we observed that cell viability and delivery efficiency in $9 \mathrm{ml} / \mathrm{h}$ had better results.

GFP expression levels ranging from $19 \%$ to $55.7 \%$ in live cells were observed approximately 24 hours posttransfection, with significant increase in expression detected in cells processed with different flow rates. (fig3 A). Interestingly, the GFP expression observed in the live cell population was linearly correlated with 
increasing flow rate until $11 \mathrm{ml} / \mathrm{h}$, indicating that the overall expression level of GFP by augmenting chaotic advection in channel has been enhanced.

While delivery efficiency and viability are important success metrics for cell engineering, unintended changes to cell phenotype may adversely impact functional potential. Accompanying the significant decrease in viability, cell death is remarkable in nucleofected cells and progresses days after transfection (Zhang et al., 2014).In comparison, intracellular delivery of gene via our design showed only slight decreases in cell viability two days after transfection compared to nucleofection. Quantifying the GFP expression levels in transfected cells revealed a peak transfection efficiency of $55.7 \%$ when applying $0.5 \mu \mathrm{g} / \mathrm{ml}$ plasmid. (fig3 B) We found that the levels of GFP expression were significantly higher across all concentrations and speeds compared to nucleofection. Cumulatively, these results suggest that delivery of plasmid DNA at various concentration and different flow rates via our microfluidic device varies the transfection efficiency and cell viability with optimal levels achieved when delivering $0.5 \mu \mathrm{g} \mathrm{mL}-1$ DNA in $9 \mathrm{ml} / \mathrm{h}$ speed.

\section{Expression of target gene has been elevated in microfluidic device}

In order to confirm the efficiency of serpentine microfluidic device, comparison of gene expression in two (nucleofection) and three-dimensional system (Microfluidic chip) 24 hours post transfection was performed by Real-Time PCR technique. The mRNA expression of anti-BCMA gene in serpentine device is 33.8 \pm 1.7 fold higher than nucleofection (fig.4A). In addition, post hoc analysis of surface protein expression differentiates delivery approaches. Based on results have been showed in fig.4B, our 3D system gene delivery approach was capable of inducing significant expression of anti- BCMA surface protein, three fold, higher than nucleofection method. Taken together, molecular consequences of nucleofection relative to new- generation delivery system highlight the importance of selected delivery strategy for both research and therapeutic applications.

\section{Discussion}

As we know, immune cells are among the key cell groups in biological studies and clinical applications but intracellular transfer of biomolecules such as DNA and siRNA to immune cells is difficult. In the case of T cells, it has been reported that RNA transfer to the cytoplasm is not difficult, but DNA transfer is difficult and challenging due to the need to cross the nucleus. In general, immune cells exhibit low tolerance and high resistance to plasmid entry regardless of gene delivery technique (Hendel et al., 2015). Electroporation is one of the techniques that works well in the transfer of SiRNA, mRNA to T lymphocytes but there are issues such as high killing, alteration of activation process, signaling pathways and expression profiles of essential genes that need to be addressed (Bhagat, Kuntaegowdanahalli, Kaval, Seliskar, \& Papautsky, 2010).

numerous published papers about electroporation all agree that there is a linear relationship between efficiency and cell death as the main challenge in cell electroporation is long-term survival and post-pulse electrical activity. According to published reports, the lack of viability, growth and proliferation after electrical pulse induction in cells is one of the major disadvantages of this method for using as gene delivery approach, especially for primary and immune cells(Lenz, Bacot, Frazier-Jessen, \& Feldman, 2003).

Concerns about efficiency and safety of various cell engineering approaches have limited cell-based therapies in the past. So far, the standards of manufacturing engineered cells for gene therapy have been with the aid of viral methods that are time consuming, laborious and expensive. In addition, cells produced by the viral method require very high safety equipment and conditions as well as follow-up and patient care for up to 15 years after treatment(Roodman, 1999).

Non-viral methods of gene transfer are a good alternative to produce engineered cells because they allow the transcellular delivery of various RNA, DNA, and macromolecules, such as proteins, temporarily or permanently. In addition, these methods have advantages over the current standard method, which can save time and cost as well as ease of scale. Physical transfection methods such as electroporation are easy, fast, efficient and $80 \%$ effective in gene transfer to T cells (Levine et al., 2017) But the low cell viability in this approach has limited the tendency for this method to be used in high-scale cell therapy that requires a large 
number of viable and active cells.

Using microfluidic knowledge is a lucrative solution to these problems that can save time and reduce concerns about virus safety and patient outcomes. This chipset designed by us is capable of producing large number of transfected cells at a designated speed without creating a clogging cell mass. In addition, according to published paper from Justin A. Jarrell, this technique had no negative effect on cell growth and viability compared to electroporation, which was less than $40 \%$ two to three days after transfection.(Bhagat et al., 2010)

Based on the percentage of cells transfected with serpentine devices, this study highlights the importance of microfluidic techniques for transferring genes to transfected- resistant blood cells.

As noted in the preceding paragraphs, the electroporation method or its improved form, nucleofection, is actively replacing method instead of viral systems for producing engineered cells. However, in addition to high levels of cell death, it results in increased cell activation, altered cellular gene expression profiles, and consequently altered cell function in the patient's body, which resulted from severe pulse-induced stress. In an electroporation-based study, the PD-1 gene was suppressed in T lymphocytes by Crisper following transfection, which increased cytokine production and resulted in altered expression of genes associated with cell proliferation and survival. surprisingly, in addition to altering the expression of these genes, there was a disruption in the expression of genes responsible for DNA repair, growth factor production, cell proliferation, and decreased cellular ability to counteract the tumor (Tchou et al., 2017).However, in a study using microfluidics for T-cell gene transfer, unlike nucleofection No changes in expression of important surface markers in survival, activity and function of $\mathrm{T}$ lymphocytes were observed.

In general, studies and results indicate that the use of microfluidic technology, unlike nucleofection methods, will not negatively affect cell viability and expression of critical genes.

The microfluidic pattern employed in this study is less efficient than the compression and constriction pattern shown in several papers on different cells, possibly due to the greater impact of membrane disruption caused by the compression created with these chipsets (Jarrell et al., 2019). However, this decrease in the delivery efficiency in the serpentine device is compensated by the high cell viability, the ability to regulate high cell concentration at low volume and the plasmid without causing clogging cell (commonly seen in constrictionbased chips) compared to constriction-based devices (Stewart, Langer, \& Jensen, 2018). In addition, it takes less time for gene transfer by this pattern, resulting in less stress following shear stress and turbulency inside the device. While both nucleofection and cell squeezing rely on membrane disruption, our study indicates that mechanical membrane penetration coupled with diffusion-mediated delivery significantly detracts unintended negative consequences. In terms of genetic factor consumption (here the plasmid), two-dimensional systems such as nucleofection as well as virus production methods require a large amount of high OD plasmids.

For example, for each nucleofection reaction according to the Lunza instructions, 2 million myeloma cells need $5 \mu \mathrm{g}$ gene, whereas in this chipset even low OD plasmids has no negative effect on outcome, because this technique unlike the routine methods used in two-dimensional systems such as chemical and nucleofectionbased methods is independent of $\mathrm{Ph}$.

Overall, our study provides a novel approach to the field of gene transfer to cells, especially transfectionresistant cells. here, we utilized a microfluidic system which can deliver plasmid DNA to myeloma cells with $55.7 \%$ efficiency. The model designed in this study, which was first applied to blood cells, consists of multiple U-shaped loops that cell is affected by the channel walls under the influence of forces such as drag and centrifugal force. The cell membrane become porous after consecutive hits, and the plasmid containing the gene enters the cell. This device has the potential to completely removes the two primary barriers to widespread gene-modified cell therapy: cost and scalability. The device may also reduce or even eliminate many of the challenges described in this paper.

In conclusion, Gene-modified cell therapies (GMCT) have brought hope for the treatment of an array of diseases. However, the development of GMCTs have been limited by issues surrounding the most critical 
step in their production, the introduction of the genetic material. In this paper, we compared nucleofection to a microfluidic transfection device and we understand that microfluidic technology had dramatically fewer side effects and higher efficiency. The significant differences in viability and rate of engineered cells underscores the importance of understanding the impact of intracellular delivery systems on cell function for research and clinical applications.

\section{Acknowledgments}

This work was performed at Tarbiat Modares Medical University. The authors would

like to acknowledge Pasteur Institute of Iran for gifting cells.

\section{References}

Aref, H. (1984). Stirring by chaotic advection. Journal of Fluid Mechanics , 143 , 1-21. https://doi.org/10.1017/S0022112084001233

Bhagat, A. A. S., Kuntaegowdanahalli, S. S., Kaval, N., Seliskar, C. J., \& Papautsky, I. (2010). Inertial microfluidics for sheath-less high-throughput flow cytometry. Biomedical Microdevices ,12 (2), 187-195. https://doi.org/10.1007/s10544-009-9374-9

Bilal, M. Y., Vacaflores, A., \& Houtman, J. C. (2015). Optimization of methods for the genetic modification of human T cells. Immunology and Cell Biology, 93 (10), 896-908. https://doi.org/10.1038/icb.2015.59

Cox, D. B. T., Platt, R. J., \& Zhang, F. (2015). Therapeutic genome editing: prospects and challenges. Nature Medicine , 21 (2), 121-131. https://doi.org/10.1038/nm.3793

Escoffre, J.-M., Portet, T., Wasungu, L., Teissié, J., Dean, D., \& Rols, M.-P. (2009). What is (Still not) Known of the Mechanism by Which Electroporation Mediates Gene Transfer and Expression in Cells and Tissues. Molecular Biotechnology , 41 (3), 286-295. https://doi.org/10.1007/s12033-008-9121-0

Franssen, L. E., Mutis, T., Lokhorst, H. M., \& van de Donk, N. W. C. J. (2019). Immunotherapy in myeloma: how far have we come? Therapeutic Advances in Hematology , 10 , 204062071882266. https://doi.org/10.1177/2040620718822660

Hendel, A., Bak, R. O., Clark, J. T., Kennedy, A. B., Ryan, D. E., Roy, S., .. Porteus, M. H. (2015). Chemically modified guide RNAs enhance CRISPR-Cas genome editing in human primary cells. Nature Biotechnology , 33 (9), 985-989. https://doi.org/10.1038/nbt.3290

Jarrell, J. A., Twite, A. A., Lau, K. H. W. J., Kashani, M. N., Lievano, A. A., Acevedo, J., ... Pawell, R. S. (2019). Intracellular delivery of mRNA to human primary T cells with microfluidic vortex shedding. Scientific Reports , 9 (1), 3214. https://doi.org/10.1038/s41598-019-40147-y

Kebriaei, P., Singh, H., Huls, M. H., Figliola, M. J., Bassett, R., Olivares, S., .. Cooper, L. J. N. (2016). Phase I trials using Sleeping Beauty to generate CD19-specific CAR T cells. Journal of Clinical Investigation , 126 (9), 3363-3376. https://doi.org/10.1172/JCI86721

Kyle, R. A., Gertz, M. A., Witzig, T. E., Lust, J. A., Lacy, M. Q., Dispenzieri, A., .. Greipp, P. R. (2003). Review of 1027 Patients With Newly Diagnosed Multiple Myeloma. Mayo Clinic Proceedings ,78 (1), 21-33. https://doi.org/10.4065/78.1.21

Lenz, P., Bacot, S. M., Frazier-Jessen, M. R., \& Feldman, G. M. (2003). Nucleoporation of dendritic cells: efficient gene transfer by electroporation into human monocyte-derived dendritic cells 1.FEBS Letters , 538 (1-3), 149-154. https://doi.org/10.1016/S0014-5793(03)00169-8

Levine, B. L., Miskin, J., Wonnacott, K., \& Keir, C. (2017). Global Manufacturing of CAR T Cell Therapy. Molecular Therapy - Methods \& Clinical Development , 4 , 92-101. https://doi.org/10.1016/j.omtm.2016.12.006 
Loessner, D., Stok, K. S., Lutolf, M. P., Hutmacher, D. W., Clements, J. A., \& Rizzi, S. C. (2010). Bioengineered 3D platform to explore cell-ECM interactions and drug resistance of epithelial ovarian cancer cells. Biomaterials , 31 (32), 8494-8506. https://doi.org/10.1016/j.biomaterials.2010.07.064

Palumbo, A., \& Anderson, K. (2011). Multiple Myeloma. New England Journal of Medicine , 364 (11), 1046-1060. https://doi.org/10.1056/NEJMra1011442

Roodman, G. D. (1999). Cell biology of the osteoclast.Experimental Hematology , 27 (8), 1229-1241. https://doi.org/10.1016/S0301-472X(99)00061-2

Rosazza, C., Haberl Meglic, S., Zumbusch, A., Rols, M.-P., \& Miklavcic, D. (2016). Gene Electrotransfer: A Mechanistic Perspective.Current Gene Therapy , 16 (2), 98-129. https://doi.org/10.2174/1566523216666160331130040

Siegel, R. L., Miller, K. D., \& Jemal, A. (2016). Cancer statistics, 2016. CA: A Cancer Journal for Clinicians , 66 (1), 7-30. https://doi.org/10.3322/caac.21332

Stewart, M. P., Langer, R., \& Jensen, K. F. (2018). Intracellular Delivery by Membrane Disruption: Mechanisms, Strategies, and Concepts.Chemical Reviews , 118 (16), 7409-7531. https://doi.org/10.1021/acs.chemrev.7b00678

Tchou, J., Zhao, Y., Levine, B. L., Zhang, P. J., Davis, M. M., Melenhorst, J. J., .. J June, C. H. (2017). Safety and Efficacy of Intratumoral Injections of Chimeric Antigen Receptor (CAR) T Cells in Metastatic Breast Cancer. Cancer Immunology Research ,5 (12), 1152-1161. https://doi.org/10.1158/2326-6066.CIR-17-0189

Wang, X., \& Rivière, I. (2016). Clinical manufacturing of CAR T cells: foundation of a promising therapy. Molecular Therapy - Oncolytics , 3 , 16015. https://doi.org/10.1038/mto.2016.15

Whitesides, G. M. (2006). The origins and the future of microfluidics.Nature , 442, 368.

Xia, D., Quan, P., Piao, H., Piao, H., Sun, S., Yin, Y., \& Cui, F. (2010). Preparation of stable nitrendipine nanosuspensions using the precipitation-ultrasonication method for enhancement of dissolution and oral bioavailability. European Journal of Pharmaceutical Sciences , 40 (4), 325-334. https://doi.org/10.1016/j.ejps.2010.04.006

Zhang, M., Ma, Z., Selliah, N., Weiss, G., Genin, A., Finkel, T. H., \& Cron, R. Q. (2014). The impact of Nucleofection (B on the activation state of primary human CD4 T cells. Journal of Immunological Methods ,408, 123-131. https://doi.org/10.1016/j.jim.2014.05.014

\section{Figure Legends}

Figure 1. Design of the microfluidic transfection device. (A) Photograph of the serpentine device. (B) Schematic of microfluidic chaotic-based delivery system used to transfect DNA into human myeloma cells. Briefly, cells and DNA were mixed in suspension, chaotic advection creating

vortices in inner wall that disrupt cell membranes to permit permeability and allow DNA to diffuse into cells. (C) simulation of velocity magnitude $(\mathrm{m} / \mathrm{s})$ in chip containing U-turn channels.

Figure 2. Microscopy of myeloma cells expressing green fluorescent protein 24 hours post transfection in 2 different gene delivery systems: (A) Non-transfected cell, (B) Nucleofection, (C) Microfluidic Transfection Device.

Figure 3. Levels of EGFP protein expression in myeloma cells compared to nucleofection. (A) Representative experiment for the percent of transfected cells following microfluidic transfection reveals high GFP expression and viability compared to nucleofection. (B) Transfection efficiency in human myeloma cells transfected with various concentrations of DNA via our microfluidic device.

Figure 4. Comparison of intracellular delivery methods in mRNA and protein expression. (A) mRNA level measured by q RT-PCR of target gene $48 \mathrm{~h}$ post transfection. The results are presented as the mean $\pm \mathrm{SD}$ 
of three different experiments, $\mathrm{p}<0.001$. (B) Representative percentage of myeloma cells that exhibit surface protein expression after microfluidic processing and nucleofection $48 \mathrm{~h}$ post transfection.
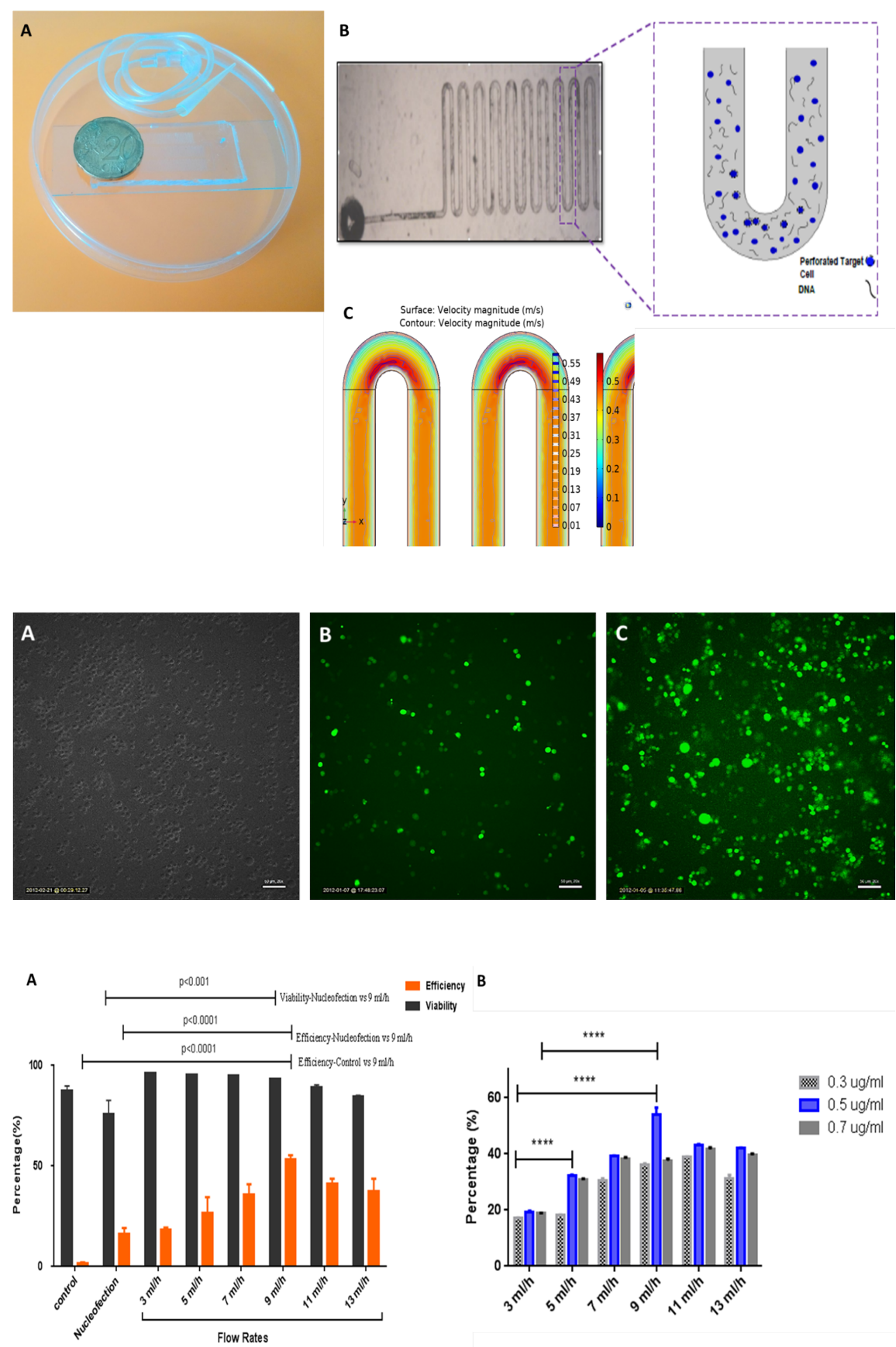

표 $0.3 \mathrm{ug} / \mathrm{ml}$

$\square 0.5 \mathrm{ug} / \mathrm{ml}$ $0.7 \mathrm{ug} / \mathrm{ml}$ 

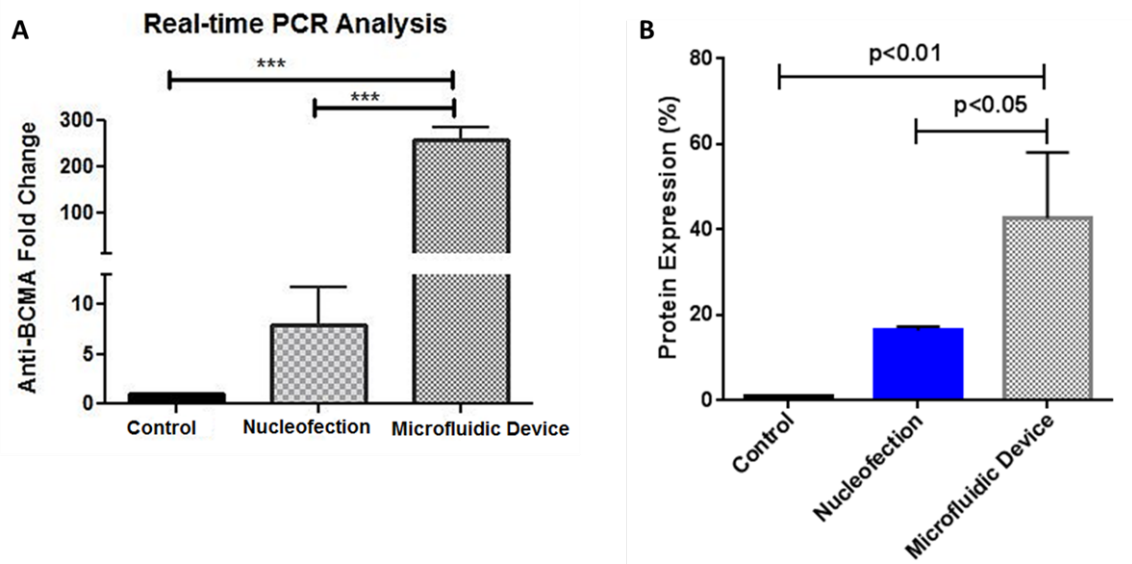